\title{
Comparative Study on Backcross Inbred Lines of IR64 Rice (Oryza sativa L.) Introgressed with Drought QTLs under Varied Moisture Regimes over Different Seasons
}

\author{
K. Baghyalakshmi ${ }^{1}$, P. Jeyaprakash ${ }^{2}$, S. Ramchander ${ }^{2}$, \\ T. Radhamani ${ }^{2}$ and M. Raveendran ${ }^{3}$
}

\author{
${ }^{1}$ Division of Crop Improvement, Central Tobacco Research Institute, Rajahmundry, \\ Andhra Pradesh, India \\ ${ }^{2}$ Centre for Plant Breeding and Genetics, Tamil Nadu Agricultural University, \\ Coimbatore --641003, Tamil Nadu, India \\ ${ }^{3}$ Department of Plant Biotechnology, Centre for Plant Molecular Biology and Biotechnology, \\ Tamil Nadu Agricultural University, --641003, Tamil Nadu, India
}

*Corresponding author

\begin{abstract}
A B S T R A C T
Among the abiotic stresses, drought is the major factor for heavy yield losses in rice production. The present study was undertaken with an aim of understanding the effect of mega QTLs of Apo controlling yield under drought. The BILs viz., CB 229 and CB 193-3 were evaluated for their drought responses under green house conditions along with the parents Apo, IR64 and check Norungan during Summer 2015, Kharif 2015, Rabi 2015-16. The results from this study reported that, Apo and BILs had higher photosynthetic rate when compared to IR64 under drought. CB 229 had low reduction per cent of photosynthesis (45.25\%), Ci/Ca $(23.88 \%)$, conductance $(52.38 \%)$ and RWC (25.72\%) compared to susceptible parent IR64. Number of filled grains was much higher in Apo, CB229 and CB 193-3 when compared to IR64 under stress condition. The significant reduction in single plant yield under moisture stress condition was observed in all the genotypes especially in IR64 (65.15\%). Reduction was relatively less in the donor Apo and BILs. CB 229 showed a greater RWC, conductance, moderate transpiration rate, increased water uptake, a higher assimilation rate and a higher grain yield under the moisture stress condition compared to the other BIL and IR64. It was found that CB 229 with three QTL, i.e., $q D T Y 2.2, q D T Y_{3.1}$ and $q D T Y_{8.1}$, showed better performance than CB 193-3 with two QTL, $q D T Y_{3.1}$ and $q D T Y_{8.1}$, under severe stress while in irrigated condition CB 229 was onpar with IR64.
\end{abstract}

Keywords

Oryza sativa,

Drought, Backcross Inbred Lines

Article Info

Accepted:

20 December 2017

Available Online:

10 January 2018

\section{Introduction}

Rice is the primary food for more than half of the world's population, especially in developing countries such as Asia, where water scarcity and drought are imminent threats to food security. Rice uses two to five times more water than other cereal food crops 
such as wheat or maize and uses about 30 per cent of the freshwater for agricultural crops worldwide. To meet the growing demand from human population which is expected to touch 9 billion by 2050, in a changing global climatic order, rice varieties with higher yield potential and greater yield stability need to be developed. Exploring ways to reduce water use for rice production is therefore of great strategic value for sustainable crop production for the world facing water scarcity (Molden $e t$ al., 2010).

Drought is the most serious environmental stress, limiting crop growth and productivity; drought induced loss in crop yield probably exceeds losses from all other causes. Water deficit is therefore a key constraint that affects rice production in different countries.

Drought tolerance is a complex trait, and a number of quantitative trait loci (QTL) for drought tolerance in rice have been identified (Hao and Lin, 2010). However, breeding drought-tolerant rice is hard to achieve by conventional strategies, including markerassisted selection. Understanding of the molecular mechanisms underlying drought tolerance is therefore needed for successful, knowledge-based crop improvement (Millan et al., 2006). Meanwhile, fundamental research has provided significant insights in the understanding of the physiological and molecular responses of plants to water deficits, but there is still a large gap between yields in optimal and stress conditions (Park et al., 2011).

Minimizing this 'yield gap' and increasing yield stability under different stress conditions are of strategic importance in guaranteeing food for the future. This could be possible reached out by studying the physiological plant response to drought and breeding drought tolerant varieties with the acquired knowledge.

\section{Materials and Methods}

This present study was conducted at Department of Rice, Centre for Plant Breeding and Genetics, Tamil Nadu Agricultural University, Coimbatore during Summer 2015, Kharif 2015, Rabi 2015-16. Backcross Inbred Lines of IR64 (four lines) developed from the cross combination of IR64 X APO $\left(\mathrm{BC}_{1} \mathrm{~F}_{5}\right)$ with different QTL combinations identified through marker assisted introgression and selection. Among them two lines namely CB 193-3 ( $q D T Y_{3.1}$ and $\left.q D T Y_{8.1}\right)$ and CB 229 $\left(q D T Y_{2.2}, q D T Y_{3.1}\right.$ and $\left.q D T Y_{8.1}\right)$ were used in the study as it showed higher tolerance to drought in previous studies (Baghyalakshmi et al., 2016). Two BILs along with the parents and Norungan a land race of Tamil Nadu tolerant to drought were used in the investigation. Apo, drought tolerant upland variety, developed at IRRI, recommended for cultivation under aerobic conditions. Owing to its drought tolerance nature and good performance under aerobic conditions, they serve as important source for mining drought tolerant QTLs. IR64 is a medium duration and high yielding variety but highly prone to drought.

\section{Drought stress treatments and measurements under greenhouse condition}

The seeds were sown and crop was raised in greenhouse during Summer 2015, Kharif 2015, Rabi 2015-16 and were subjected to drought stress along with irrigated control in four replications each. The seed materials were grown in plastic pots $(30 \mathrm{~cm}$ height $\mathrm{x} 30$ $\mathrm{cm}$ diameter with drainage hole) filled with three parts of coir pith and one part of natural clay loam soil. Three plants per pots were maintained and were grown in green house under natural temperature. The crop was irrigated till $45^{\text {th }}$ day and there after irrigation was stopped for two replication (till the Relative Water Content reached 50\%) and the 
yield parameters were recorded. The positions of the pots were changed frequently to minimize the micro climate effects. The physiological and yield parameters were recorded to select the genotype showing better performance under drought for further studies.

\section{Measurements of photosynthetic parameters and leaf osmotic potential}

Infrared Gas Analyzer (IRGA) is a portable photosynthetic system (LICOR- Model LI 6400 version.5) and major component used for the measurement of different parameters viz., photosynthetic rate, stomatal conductance, transpiration rate and $\mathrm{Ci} / \mathrm{Ca}$ ratio. The basic principle (Barrs and Weatherley, 1962) of this technique consists essentially in comparing the water content of leaf tissue when fresh leaf sampled with the fully turgid water content and expressing the results on percentage basis. Relative water content was estimated by Weatherley (1950) method and expressed in percentage. Several biometrical traits were also observed in both under control and moisture stress condition.

To measure the osmotic potential the penultimate fully expanded leaf on the main stem or on a primary tiller was cut, wrapped in a plastic bag and freezed. Further the leaf was soaked in water for $24 \mathrm{~h}$ to rehydrate the tissue. The sap was collected by squeezing the leaf sample with the help of a sterile syringe and the osmolality $\left(\mathrm{mmol} \mathrm{kg}{ }^{-1}\right)$ of the expressed sap was determined using a vapour pressure osmometer (Vapro, Model 5520 Wescor Inc., Logan, UT, USA).

Osmoticpotential (wp) was calculated as

$\Psi \pi=-\mathrm{cRT}$,

Where $\mathrm{c}$ is concentration, $\mathrm{R}$ is the universal gas constant $(0.0832)$ and $\mathrm{T}$ is the temperature in degrees Kelvin $(310 \mathrm{~K})$.
The following conversion equation was used to compute osmotic potential (in MPa)

$\left[\left(\# \mathrm{mmol} \mathrm{kg}{ }^{-1}\right)(0.0832)(310)\right] / 10000$.

Leaf rolling was determined based on a standard chart presented by O'Toole and Cruz (1980). A visual score was taken of the degree of leaf rolling as made on the sample leaf using a 1 to 5 scale with 1 being the first evidence of rolling and 5 being a closed cylinder. Plants under normal irrigation in the same period were used as controls.

\section{Protein content}

Fresh leaf sample $0.5 \mathrm{~g}$ was grounded with 5 $\mathrm{ml} 0.2 \mathrm{M}$ phosphate buffer $(\mathrm{pH}$ 7.0). The extract was centrifuged at $12,000 \mathrm{rpm}$ for 10 minutes. The pellet was dissolved in $5 \mathrm{ml}$ of $0.1 \mathrm{~N} \mathrm{NaOH}$ and the same was used to quantify proteins. The soluble protein in the rice leaf extract was determined by the method proposed by Bradford (1976) with slight modification.

Protein quantity in the sample extract was determined by pipetting out $0.2 \mathrm{ml}$ of the extract into a tube containing $0.8 \mathrm{ml}$ of $\mathrm{NaOH}$ and $5.0 \mathrm{ml}$ of Bradford dye solution and allowed to stand for 5 minutes. Absorbance was measured at $595 \mathrm{~nm}$ against the zero setting blank of $0.1 \mathrm{~N} \mathrm{NaOH}$. A sample blank was maintained essentially except adding the sample extract. The amount of protein was calculated from the standard graph prepared using bovine serum albumin fraction 5 ranging from $10-100 \mu \mathrm{g}$.

\section{Statistical analysis}

The physiological results were exported to SPSS Version 13.0 (Lead Technologies, USA) and the pooled ANOVA was done for statistical analysis. 


\section{Results and Discussion}

\section{Pooled analysis of variance}

Significant difference was observed among the genotypes studied under both stress (drought) and irrigated condition for most of the traits carbon assimilation, transpiration rate, relative water content, plant height, productive tillers, days to fifty per cent flowering, panicle length, fertile grains per panicle, fertility per cent, SPAD, total protein content, osmotic potential and single plant yield.

Some traits especially physiological traits shown difference in their performance between seasons whereas most of the phenotypic traits were stable among the environments (seasons) and this exhibited that there was no such variations among seasons under green house condition limited G x E (Genotype $\mathrm{x}$ Environment) interactions (Table 1 and 2).

\section{Performance of backcross inbred lines under irrigated and moisture stress condition}

The phenotypic changes in drought-stressed IR64, Apo, Norungan and BILs were summarized in the table 3 and 4 .

Severe drought stress was imposed at peak vegetative stage. All the five genotypes viz., IR64, Apo, CB 229 and CB 193-3 were subjected to same intensity of stress by allowing the soil moisture in pots to reach 15 - 16 per cent. Upon drought, the BIL CB 229 showed delayed leaf rolling symptom when compared to susceptible parent IR64. The tolerant BIL CB 229 did not shown any leaf rolling symptom with the SES score of zero (leaves healthy), Apo, Norungan and CB 1933 exhibited leaf rolling symptom with the SES score of 1 (leaf starts to fold) and IR64 had the symptom having the SES score of 9 (leaves tightly rolled) on 35 DASI. The BIL CB 229 and Apo were able to maintain higher relative water content $(\approx 20 \%)$ over a period of drought imposition than IR64.

At same level of drought stress i.e. $\approx 16$ per cent of soil moisture content, RWC of IR64 reduced to 45.38 per cent whereas the tolerant lines viz., Apo, CB 229 and CB 193-3 maintained 70.54 per cent, 71.63 per cent and 61.44 per cent of RWC in leaves respectively. Plant height was obviously reduced by the drought stress compared to the well-watered plants, indicating that Apo and CB-229 had better growth in leaf and stem elongation under the drought stress than IR64. The tolerant genotype CB 229 recorded higher photosynthetic rate $\left(15.61 \mathrm{mmol} \mathrm{CO}_{2} \mathrm{~m}^{-2} \mathrm{~s}^{-1}\right)$ and conductance $\left(0.20 \mathrm{mmol} \mathrm{H}_{2} \mathrm{O} \mathrm{m} \mathrm{m}^{-2} \mathrm{~s}^{-1}\right)$ when compared to the susceptible genotype IR64 which recorded photosynthetic rate of $7.27 \mathrm{mmol} \mathrm{CO}_{2} \mathrm{~m}^{-2} \mathrm{~s}^{-1}$ and conductance of $\left.0.07 \mathrm{mmol} \mathrm{H}_{2}\right) \mathrm{m}^{-2} \mathrm{~s}^{-1}$.

CB 229 had low reduction per cent of photosynthesis $(45.25 \%), \mathrm{Ci} / \mathrm{Ca}(23.88 \%)$, conductance $(52.38 \%)$ and RWC (25.72\%) than IR64. These results indicated that Apo and the BILs had more capability to cope with the drought stress than IR64.

All the plants were re-watered on $40^{\text {th }}$ day after imposition of drought stress. After rewatering, tolerant lines were able to revive when compared to susceptible genotype. Fertility per cent of IR64 was 50.54 under stress condition whereas tolerant lines Apo and CB 229 had recorded the fertility per cent of 70.75 and 66.54 respectively. Further, the grain yield was found to be higher in CB 229 $(14.13 \mathrm{~g})$ than the tolerant parent Apo (12.80 g). Thus, fertility per cent and single plant yield were found to be affected more severely due to non-availability of moisture. 
Table.1 Pooled ANOVA for different physiological traits observed during Summer 2015 (E1), Kharif 2015 (E2), Rabi $2015-16$ (E3) under controlled (irrigated) condition and drought condition

\begin{tabular}{|c|c|c|c|c|c|c|c|c|c|c|c|c|c|}
\hline \multirow[t]{2}{*}{ Source } & \multirow[t]{2}{*}{ Condition } & \multirow[t]{2}{*}{ df } & \multicolumn{11}{|c|}{ MSS } \\
\hline & & & PH & PT & DF & PL & FG & FP & CHA & TP & $\mathbf{O P}$ & HGW & SPY \\
\hline \multirow[t]{2}{*}{ Replication } & Control & \multirow[t]{2}{*}{9} & 3.09 & 2.38 & 4.41 & 0.19 & 14.01 & 1.41 & 3.84 & 0.26 & - & 0.00 & 0.08 \\
\hline & Stress & & 7.05 & 1.55 & 2.66 & 0.36 & 45.44 & 0.59 & 0.92 & 0.02 & 0.00 & 0.00 & 0.26 \\
\hline \multirow[t]{2}{*}{ Treatment } & Control & \multirow[t]{2}{*}{4} & $12339.55^{*}$ & $177.41 *$ & $343.91 *$ & $8.19 *$ & $15670.11^{*}$ & $29.69 *$ & $13.70 *$ & $11.69 *$ & - & 0.35 & $73.64 *$ \\
\hline & Stress & & $3672.09 *$ & $44.90 *$ & $325.79 *$ & $16.94 *$ & $8851.24 *$ & $736.77 *$ & $101.81 *$ & $107.75 *$ & $3.27 *$ & 0.53 & $48.72 *$ \\
\hline \multirow[t]{2}{*}{ Season } & Control & \multirow[t]{2}{*}{2} & $24.29 *$ & $159.50 *$ & $9.90 *$ & 0.11 & $142.63 *$ & 1.74 & $11.77^{*}$ & $901.57 *$ & - & 0.01 & 1.27 \\
\hline & Stress & & $20.06^{*}$ & $7.32 *$ & 2.57 & 1.00 & $145.95 *$ & 2.23 & 2.73 & $16.08^{*}$ & 1.61 & 0.01 & $4.52 *$ \\
\hline \multirow[t]{2}{*}{ SxT } & Control & \multirow[t]{2}{*}{8} & $6.96 *$ & $22.80 *$ & 2.09 & $5.26^{*}$ & $688.99 *$ & $5.56^{*}$ & $5.04 *$ & $23.52 *$ & - & 0.00 & 1.82 \\
\hline & Stress & & $10.88^{*}$ & $4.54^{*}$ & $3.17 *$ & $5.50 *$ & $60.27 *$ & $9.22 *$ & 1.73 & $2.21 *$ & 0.14 & 0.01 & 0.81 \\
\hline \multirow[t]{2}{*}{ Error } & Control & 26 & 4.80 & 0.92 & 2.14 & 0.60 & 70.52 & 1.08 & 2.21 & 0.26 & - & 0.00 & 0.33 \\
\hline & Stress & & 3.79 & 0.81 & 1.72 & 0.23 & 15.06 & 1.82 & 0.52 & 0.04 & 0.00 & 0.00 & 0.18 \\
\hline
\end{tabular}

Table.2 Pooled ANOVA for different morphological and biochemical traits observed during Summer 2015 (E1), Kharif 2015 (E2), Rabi 2015-16 (E3) under controlled (irrigated) condition and drought condition

\begin{tabular}{|c|c|c|c|c|c|c|c|c|c|c|c|c|c|c|c|c|c|}
\hline \multirow[t]{2}{*}{ Source } & \multirow[t]{2}{*}{ Condition } & \multirow[t]{2}{*}{ df } & \multicolumn{15}{|c|}{ MSS } \\
\hline & & & $\begin{array}{c}\text { PR } \\
\text { @ } \\
\text { 10DAS }\end{array}$ & $\begin{array}{c}\text { PR } \\
\text { @ } \\
\text { 25DAS }\end{array}$ & $\begin{array}{c}\text { PR } \\
\text { @ } \\
\text { 35DAS }\end{array}$ & $\begin{array}{c}\text { CI/CA } \\
@ \\
\text { 10DAS }\end{array}$ & $\begin{array}{c}\text { CI/CA } \\
\text { @ } \\
\text { 25DAS }\end{array}$ & $\begin{array}{c}\text { CI/CA } \\
@ \\
\text { 35DAS }\end{array}$ & $\begin{array}{c}\text { CON } \\
\text { @ } \\
\text { 10DAS }\end{array}$ & $\begin{array}{c}\text { CON } \\
@ \\
\text { 25DAS }\end{array}$ & $\begin{array}{c}\text { CON } \\
\text { @ } \\
\text { 35DAS }\end{array}$ & $\begin{array}{c}\text { TR } \\
@ \\
\text { 10DAS }\end{array}$ & $\begin{array}{c}\text { TR } \\
@ \\
\text { 25DAS }\end{array}$ & $\begin{array}{c}\text { TR } \\
\text { @ } \\
\text { 35DAS }\end{array}$ & $\begin{array}{c}\text { RWC } \\
\text { @ } \\
\text { 10DAS }\end{array}$ & $\begin{array}{c}\text { RWC } \\
@ \\
\text { 25DAS }\end{array}$ & $\begin{array}{c}\text { RWC } \\
\text { @ 35DAS }\end{array}$ \\
\hline \multirow[t]{2}{*}{ Replication } & C & 9 & 0.27 & 0.47 & 0.25 & 0.00 & 0.00 & 0.00 & 0.00 & 0.00 & 0.00 & 0.06 & 0.09 & 0.03 & 5.30 & 4.56 & 2.49 \\
\hline & S & & 0.20 & 0.12 & 0.05 & 0.00002 & 0.00002 & 0.00002 & 0.00002 & & 0.00002 & 0.08 & 0.03 & 0.01 & 2.75 & 5.46 & 1.32 \\
\hline \multirow[t]{2}{*}{ Treatment } & $\mathrm{C}$ & 4 & $45.66^{*}$ & $39.66^{*}$ & $3.28 *$ & 0.01 & 0.00 & 0.02 & 0.25 & 0.01 & 0.02 & $4.08^{*}$ & 1.28 & $9.62^{*}$ & $28.91^{*}$ & $33.32 *$ & $7.34 *$ \\
\hline & S & & $26.95 *$ & $39.17^{*}$ & $152.23^{*}$ & 0.01 & 0.01 & 0.03 & 0.00 & & 0.05 & $6.90^{*}$ & $8.04 *$ & $28.99^{*}$ & $4.91 *$ & $97.02 *$ & $1505.30^{*}$ \\
\hline \multirow[t]{2}{*}{ Season } & $\mathrm{C}$ & 2 & $169.47 *$ & $48.77 *$ & $1024.04 *$ & 0.07 & 0.13 & 0.18 & 0.39 & 0.52 & 0.35 & $80.47 *$ & $80.89^{*}$ & $257.69^{*}$ & $70.83^{*}$ & $18.12^{*}$ & $14.18^{*}$ \\
\hline & S & & $493.91 *$ & $146.53 *$ & $134.37 *$ & 0.50 & 0.27 & 0.08 & 0.07 & & 0.73 & $465.58^{*}$ & $292.74 *$ & $89.44 *$ & $9.14^{*}$ & $286.34 *$ & $2812.78^{*}$ \\
\hline \multirow[t]{2}{*}{ SxT } & $\mathrm{C}$ & 8 & $31.57 *$ & $11.09^{*}$ & $24.19 *$ & 0.01 & 0.00 & 0.01 & 0.01 & 0.01 & 0.03 & $27.42 *$ & 0.99 & $7.17^{*}$ & $24.17 *$ & $15.39^{*}$ & $11.16^{*}$ \\
\hline & S & & $9.54^{*}$ & $14.95^{*}$ & $14.90^{*}$ & 0.01 & 0.01 & 0.01 & 0.00 & & 0.03 & $4.11^{*}$ & $8.43^{*}$ & $8.98^{*}$ & $3.32 *$ & $23.87^{*}$ & $308.77 *$ \\
\hline \multirow[t]{2}{*}{ Error } & $\mathrm{C}$ & 26 & 0.36 & 0.36 & 0.34 & 0.00 & 0.00 & 0.00 & 0.00 & 0.00 & 0.00 & 0.10 & 0.08 & 0.10 & 4.29 & 1.76 & 1.24 \\
\hline & S & & 0.17 & 0.09 & 0.03 & 0.00001 & 0.00001 & 0.00001 & 0.00001 & & 0.00001 & 0.05 & 0.04 & 0.01 & 2.68 & 1.48 & 1.35 \\
\hline
\end{tabular}




\section{Int.J.Curr.Microbiol.App.Sci (2018) 7(1): 2716-2725}

Table.3 Mean performance of BILs and parents for various morphological and biochemical traits under irrigated and drought condition during Summer 2015 (E1), Kharif 2015 (E2), Rabi 2015-16 (E3)

\begin{tabular}{|c|c|c|c|c|c|c|c|c|c|c|c|c|c|c|c|c|c|c|c|c|c|c|}
\hline \multirow[t]{2}{*}{ Genotype } & \multicolumn{2}{|c|}{$\mathrm{DF}$} & \multicolumn{2}{|c|}{$\mathrm{PH}$} & \multicolumn{2}{|c|}{ PT } & \multicolumn{2}{|c|}{ PL } & \multicolumn{2}{|c|}{ FG } & \multicolumn{2}{|c|}{ FP } & \multicolumn{2}{|c|}{ HGW } & \multicolumn{2}{|c|}{ SPY } & \multicolumn{2}{|c|}{ OP } & \multicolumn{2}{|c|}{ TP } & \multicolumn{2}{|c|}{ CHA } \\
\hline & C & $\mathbf{S}$ & C & $\mathbf{S}$ & C & $\mathbf{S}$ & C & $\mathbf{S}$ & C & $\mathbf{S}$ & C & $\mathbf{S}$ & C & $\mathbf{S}$ & C & $\mathbf{S}$ & C & $\mathbf{S}$ & C & $\mathbf{S}$ & C & $\mathbf{S}$ \\
\hline IR64 & 81.29 & 73.54 & $96.68 * *$ & $80.73 *$ & $16.07^{* *}$ & 9.15 & 25.36 & 22.40 & 173.99 & 91.62 & $91.27 * *$ & 50.54 & $2.78^{* *}$ & 2.11 & $25.28 * *$ & 8.81 & - & 0.46 & 18.17 & 6.37 & 45.74 & 35.50 \\
\hline Apo & 73.01 ** & $67.11 *$ & 112.10 & 88.75 & 9.48 & 7.30 & 26.49 & 24.77 & $244.00^{* * *}$ & $166.83^{* *}$ & 90.70 & $70.75 * *$ & 2.36 & 2.23 & 19.28 & $12.80^{* * *}$ & - & $1.61 * *$ & 19.97 & $12.27^{* * *}$ & 43.80 & 42.61 ** \\
\hline CB 229 & $77.37 *$ & $66.13 * *$ & $75.86^{* *}$ & $64.55^{* *}$ & $18.46^{* * *}$ & $12.38 * *$ & $27.67 * *$ & 25.39 ** & $237.65^{* *}$ & 138.39 ** & 90.46 & $66.54 * *$ & 2.67 & $2.46 * *$ & $24.89 * *$ & $14.13^{* * *}$ & - & $1.64 * *$ & $20.70^{* *}$ & $13.73^{* *}$ & $46.43^{*}$ & $42.30 * *$ \\
\hline CB 193-3 & $74.62 * *$ & $62.98 * *$ & $77.13^{* * *}$ & 69.03 ** & $17.74 * *$ & $10.95^{* *}$ & 26.80 & 24.48 & 175.24 & 122.96 & 90.31 & $65.73 * *$ & 2.64 & $2.44 * *$ & $24.03 * *$ & 11.84 & - & $1.42 * *$ & $20.1^{*}$ & $13.30^{* *}$ & 44.95 & $41.45^{* *}$ \\
\hline Norungan & 86.27 & 75.31 & 153.84 & 108.55 & 12.11 & 9.24 & 26.63 & $25.14 * *$ & 175.74 & 131.11 & 87.27 & $67.10^{* *}$ & $2.77 * *$ & $2.64 * *$ & 22.03 & $12.96 * *$ & - & $1.74 * *$ & 19.10 & $12.4 * *$ & 44.28 & 41.10 \\
\hline Grand mean & 78.51 & 69.01 & 103.12 & 82.32 & 14.77 & 9.81 & 26.59 & 24.44 & 201.32 & 130.18 & 90.00 & 64.13 & 2.64 & 2.37 & 23.10 & 12.11 & - & 1.37 & 19.61 & 11.61 & 45.04 & 40.59 \\
\hline CD@5\% & 1.20 & 1.08 & 1.80 & 1.60 & 0.79 & 0.74 & 0.63 & 0.40 & 6.89 & 3.18 & 0.85 & 1.11 & 0.04 & 0.04 & 0.47 & 0.35 & - & 0.03 & 0.41 & 0.16 & 1.22 & 0.59 \\
\hline CD@1\% & 1.60 & 1.43 & 2.40 & 2.13 & 1.05 & 0.99 & 0.85 & 0.53 & 9.19 & 4.25 & 1.14 & 1.47 & 0.05 & 0.05 & 0.63 & 0.47 & - & 0.04 & 0.55 & 0.22 & 1.63 & 0.79 \\
\hline \multicolumn{23}{|c|}{ Environment } \\
\hline E1 & 79.30 & 69.40 & 104.30 & 82.61 & 14.16 & 10.25 & 26.61 & 24.36 & 198.41 & 132.15 & 89.90 & 64.47 & 2.66 & 2.39 & 23.39 & $12.49^{* *}$ & - & 1.20 & $24.98^{* * *}$ & 11.32 & 45.73 & 40.93 \\
\hline E2 & 78.28 & 68.69 & 102.12 & 81.21 & 12.30 & 9.12 & 26.51 & 24.69 & 201.91 & 131.28 & 90.33 & 63.79 & 2.64 & 2.35 & 22.94 & 11.57 & - & 1.22 & 12.08 & $12.62^{* *}$ & 45.17 & 40.19 \\
\hline E3 & 77.95 & 68.95 & 102.95 & 83.15 & $17.85^{* * *}$ & 10.05 & 26.65 & 24.26 & 203.65 & 127.10 & 89.77 & 64.14 & 2.63 & 2.38 & 22.97 & 12.26 & - & $1.7 * *$ & $21.76 *$ & 10.90 & 44.22 & 40.64 \\
\hline G M & 78.51 & 69.01 & 103.12 & 82.32 & 14.77 & 9.81 & 26.59 & 24.44 & 201.32 & 130.18 & 90.00 & 64.13 & 2.64 & 2.37 & 23.10 & 12.11 & - & 1.37 & 19.61 & 11.61 & 45.04 & 40.59 \\
\hline CD@5\% & 0.93 & 0.83 & 1.39 & 1.24 & 0.61 & 0.57 & 0.49 & 0.31 & 5.34 & 2.47 & 0.66 & 0.86 & 0.03 & 0.03 & 0.37 & 0.27 & - & 0.02 & 0.32 & 0.13 & 0.95 & 0.46 \\
\hline $\mathrm{CD} @ 1 \%$ & 1.24 & 1.11 & 1.86 & 1.65 & 0.81 & 0.76 & 0.66 & 0.41 & 7.12 & 3.29 & 0.88 & 1.14 & 0.04 & 0.04 & 0.49 & 0.36 & - & 0.03 & 0.43 & 0.17 & 1.26 & 0.61 \\
\hline
\end{tabular}

C- Control, S- Stress 
Table.4 Mean performance of BILs and parents for various physiological traits under irrigated and drought condition observed during Summer 2015 (E1), Kharif 2015 (E2), Rabi 2015-16 (E3)

\begin{tabular}{|c|c|c|c|c|c|c|c|c|c|c|c|c|c|c|c|c|c|c|c|c|}
\hline \multirow[t]{2}{*}{ Genotype } & \multicolumn{2}{|c|}{ PR@10DASI } & \multicolumn{2}{|c|}{ PR@35DASI } & \multicolumn{2}{|c|}{ CI/CA@10DASI } & \multicolumn{2}{|c|}{ CI/CA@35DASI } & \multicolumn{2}{|c|}{ CON@ 10DASI } & \multicolumn{2}{|c|}{ CON@ @ 35DASI } & \multicolumn{2}{|c|}{ TR @ 10DASI } & \multicolumn{2}{|c|}{ TR@ @35DASI } & \multicolumn{2}{|c|}{ RWC@10DASI } & \multicolumn{2}{|c|}{ RWC @ 35DASI } \\
\hline & C & $\mathbf{S}$ & C & $\mathbf{S}$ & C & $\mathbf{S}$ & $\mathbf{C}$ & $\mathbf{S}$ & C & $\mathbf{S}$ & C & $\mathbf{S}$ & C & $\mathbf{S}$ & C & $\mathbf{S}$ & C & $\mathbf{S}$ & C & $\mathbf{S}$ \\
\hline IR64 & 28.22 & 21.64 & 28.40 & 7.27 & 0.62 & $0.67 * *$ & $0.78 * *$ & 0.35 & 0.41 & $0.39 * *$ & $0.53 *$ & 0.07 & 14.43 & $9.70 * *$ & 15.80 & $2.91 * *$ & 98.05 & 96.63 & 96.75 & 45.38 \\
\hline Apo & 26.68 & $25.10^{* *}$ & 28.59 & $14.05 * *$ & 0.62 & 0.62 & $0.73^{*}$ & 0.45 & 0.40 & 0.35 & 0.46 & $0.16 * *$ & 14.03 & 10.98 & $13.35^{* * *}$ & 5.98 & 97.80 & 97.42 & 97.61 & $71.54 * *$ \\
\hline CB 229 & $28.96^{* *}$ & $24.51^{* *}$ & 28.51 & $15.61 * *$ & 0.62 & 0.64 & 0.67 & $0.51 * *$ & 0.41 & 0.34 & 0.42 & $0.20 * *$ & 14.72 & 11.16 & $14.32 *$ & 5.22 & 94.45 & 97.68 & 96.43 & $71.63 * *$ \\
\hline CB 193-3 & $30.94 *$ & 22.35 & 28.71 & $12.66^{* * *}$ & $0.67 * *$ & 0.64 & 0.69 & $0.46^{*}$ & $0.72 * *$ & $0.36^{*}$ & 0.43 & $0.17 * *$ & $13.35^{* *}$ & 11.48 & 14.93 & 6.47 & 96.39 & 96.32 & 95.43 & 61.44 \\
\hline Norungan & 26.00 & $24.30^{* *}$ & $29.69 * *$ & 8.62 & 0.61 & $0.65 *$ & $0.73 *$ & $0.50 * *$ & 0.38 & 0.33 & $0.49 * *$ & 0.09 & $13.51 * *$ & 11.60 & 14.58 & $3.47 * *$ & 95.33 & 96.31 & 96.67 & 55.22 \\
\hline Grand mean & 28.16 & 23.58 & 28.78 & 11.64 & 0.63 & 0.64 & 0.72 & 0.45 & 0.46 & 0.35 & 0.47 & 0.14 & 14.01 & 10.98 & 14.60 & 4.81 & 96.40 & 96.87 & 96.57 & 61.04 \\
\hline CD@5\% & 0.49 & 0.34 & 0.48 & 0.15 & 0.01 & 0.01 & 0.01 & 0.01 & 0.01 & 0.01 & 0.01 & 0.00 & 0.26 & 0.19 & 0.25 & 0.06 & 1.70 & 1.34 & 0.91 & 0.95 \\
\hline CD@1\% & 0.66 & 0.45 & 0.63 & 0.20 & 0.01 & 0.01 & 0.01 & 0.01 & 0.01 & 0.01 & 0.01 & 0.00 & 0.34 & 0.25 & 0.34 & 0.08 & 2.27 & 1.79 & 1.22 & 1.27 \\
\hline \multicolumn{21}{|c|}{ Environment } \\
\hline E1 & $28.95 * *$ & $28.11 * *$ & $34.21 * *$ & $13.14 * *$ & $0.65 * *$ & $0.67 * *$ & $0.83^{* *}$ & $0.54 * *$ & $0.55^{* *}$ & $0.50^{* * *}$ & $0.55^{* *}$ & $0.14 *$ & $14.75^{* *}$ & $15.14 * *$ & $17.87 * *$ & $5.70 * *$ & $98.33^{* *}$ & 97.63 & 96.10 & 48.10 \\
\hline E2 & 24.93 & 18.26 & 20.67 & 8.65 & 0.56 & 0.58 & 0.65 & 0.23 & 0.30 & 0.19 & 0.31 & 0.07 & 11.74 & 5.69 & 10.76 & 2.39 & 96.32 & 96.32 & $97.55^{* *}$ & $63.66 * *$ \\
\hline E3 & 30.59 ** & $24.36^{* * *}$ & $31.46 * *$ & $13.14 * *$ & $0.68 * *$ & $0.69 * *$ & 0.67 & $0.59 * *$ & $0.54 * *$ & $0.37 * *$ & $0.54 * *$ & $0.20 * *$ & $15.54 * *$ & $12.13 * *$ & $15.16^{* * *}$ & $6.33 * *$ & 94.56 & 96.67 & $96.08 * *$ & $71.38 * *$ \\
\hline G M & 28.16 & 23.58 & 28.78 & 11.64 & 0.63 & 0.64 & 0.72 & 0.45 & 0.46 & 0.35 & 0.47 & 0.14 & 14.01 & 10.98 & 14.60 & 4.81 & 96.40 & 96.87 & 96.57 & 61.04 \\
\hline CD@5\% & 0.38 & 0.26 & 0.37 & 0.12 & 0.01 & 0.01 & 0.01 & 0.01 & 0.01 & 0.00 & 0.01 & 0.00 & 0.20 & 0.15 & 0.20 & 0.05 & 1.32 & 1.04 & 0.71 & 0.74 \\
\hline CD@1\% & 0.51 & 0.35 & 0.49 & 0.16 & 0.01 & 0.01 & 0.01 & 0.01 & 0.01 & 0.01 & 0.01 & 0.00 & 0.26 & 0.20 & 0.26 & 0.06 & 1.76 & 1.39 & 0.94 & 0.98 \\
\hline
\end{tabular}

C- Control, S- Stress

\begin{tabular}{|l|l|}
\hline DF- Days to fifty per cent flowering & OP- Osmotic potential \\
PH- Plant height $(\mathrm{cm})$ & TSP -Total soluble protein \\
PT- Number of productive tillers per plant & CHA- SPAD \\
PL- Panicle length $(\mathrm{cm})$ & PR1, PR2- Carbon Assimilation rate \\
FG- Number of fertile grains per panicle & CON1, CON2 -Conductance \\
FP -Fertility percentage & TR1, TR2 -Transpiration rate \\
HGW -Hundred Grain weight $(\mathrm{g})$ & CI/CA1, CI/CA2 - Ci/Ca ratio \\
SPY- Single plant yield (g) & RWC1, RWC2- Relative leaf water content $(\%)$ \\
\hline
\end{tabular}


Evaluation of yield and physiological parameters under severe moisture stress in green house condition

Under stress condition, the genotypes varied widely for all the physiological traits studied viz., photosynthetic rate, transpiration rate, stomatal conductance and relative water content. Leaf rolling symptom was observed very early in the susceptible genotype compared to CB 229 and Apo. Rolling rapidly reduces effective leaf area and transpiration, and thus is a useful drought-avoidance mechanism in arid areas (Clarke, 1982). The increase in leaf photosynthetic rate is important to increase the yield potential of rice (Hirasawa et al., 2010) because the photosynthetic rate of individual leaves which form the canopy, affect dry matter production. Drought stress slows down carbohydrate synthesis and/or weakens the sink strength at reproductive stages and abortion of fertilized ovaries (Rahman et al., 2002).

Stomata play a paramount role in the control of water loss and gas exchange in leaves. During the onset of drought, stomatal conductivity declines before photosynthesis, and the inhibition of photosynthesis during mild stress is mainly due to the reduction of $\mathrm{CO}_{2}$ diffusion (Lawlor, 2002). However, the appearance of non-stomatal limitation to photosynthesis was evident in the drought tolerant lines as deduced from an increase in $\mathrm{Ci} / \mathrm{Ca}$ ratio. In this study, Apo and BILs had higher photosynthetic rate when compared to IR64. CB 229 had low reduction per cent of photosynthesis (45.25\%), ci/ca (23.88\%), conductance $(52.38 \%)$ and RWC $(25.72 \%)$ compared to susceptible parent IR64. Apo had higher stomatal conductance when compared to IR64. BILs also had higher stomatal conductance than IR64 among which CB 229 performed better than CB 193-3. Same kind of results were obtained by Tezera et al., (2002) who reported that higher stomatal conductance would result in higher photosynthetic rate and biomass production. This was in agreement with the results of study by Beena et al., (2012) where they have reported that water stress tolerant rice genotypes had comparatively higher protein content than susceptible lines under water stress condition. Martinez et al., (2007) also pointed out that higher stomatal conductance may be an enhanced adaptation of plants to drought environments. Araus et al., (2002) reported that higher yielding genotypes under drought had greater stomatal conductance and transpiration rate. Sikuku et al., (2010) observed transpiration rate in NERICA rice varieties generally decreased with increase in soil water deficit.

CB 229 had higher carbon assimilation rate when compared to CB193-3 under drought stress and was on par with IR 64 under controlled condition. Plant growth is determined by the ratio between photosynthetic $\mathrm{CO}_{2}$ assimilation and respiratory $\mathrm{CO}_{2}$ release. The rate of respiration is regulated by processes that use the respiratory products - ATP (water and solute uptake by roots, translocation of assimilates to sink tissues), NADH and TCA cycle intermediates (biosynthetic processes in growing parts of a plant), which together contribute to plant growth. Under moisture stress, these processes are affected and result in a decreased respiration rate. A significant difference in relative water content (RWC) was observed among genotypes between drought stress and irrigated condition. In water stress condition, higher value of RWC was recorded in stress tolerant rice genotypes as compared to susceptible genotypes at reproductive stage. This was in agreement with the results of Jha and Singh (1997) and Beena et al., (2012).

In general IR64 had higher number of tillers when compared to that of Apo, but the filled grains were much reduced whereas Apo, CB229 and CB 193-3 were recorder higher 
number of filled grains per panicle under stress condition. Similar kinds of reports regarding yield reduction in severe moisture stress are available in earlier works (Atlin et al., 2004; Venuprasad et al., 2007). Liu et al., (2005) reported that spikelet fertility reduced due to the incidence of water stress and thereby reducing the grain yield. Results of this study also indicated that the susceptible parent (IR64) had high spikelet sterility percentage when compared to drought tolerant parent Apo and the BILs.Narrow difference was observed for mean values of panicle length under moisture stress and irrigated condition. The significant reduction in single plant yield under moisture stress condition was observed in all the genotypes namely IR64 (65.15\%), Apo (33.61\%), CB 229 (43.23\%), CB 193-3 (50.73\%) and Norungan $(47.58 \%)$. This revealed that the reduction in grain yield was lower in CB 229 when compared to the susceptible genotype IR64. This indicates that drought stress during the reproductive period affects assimilate translocation from leaf to grain, via altering source-sink relationships. The reduction in leaf cell expansion would decrease sink strength for vegetative growth and lessen the competition with panicle growth for assimilates. This effect might be due to a decrease in translocation of assimilates towards reproductive organs (Hsiao and $\mathrm{Xu}$, 2000). It is important to note that all the QTL region increase grain yield under stress conditions and did not have any effect on grain yield under non stress condition and showed grain yield on par with IR64 under irrigated condition. However $q D T Y_{2.2}$, $q D T Y_{3.1}$ and $q D T Y_{8.1}$ has shown a high consistent additive effect under severe drought.CB 229 showed a greater RWC, conductance, moderate transpiration rate, increased water uptake, a higher assimilation rate and a higher grain yield under the moisture stress condition compared to the other BIL and IR64. It was found that CB 229 with three QTL, i.e., $q D T Y_{2.2}, q D T Y_{3.1}$ and $q D T Y_{8.1}$, showed better performance than CB 193-3 with two QTL, $q D T Y_{3.1}$ and $q D T Y_{8.1}$, under severe stress. In the present study, after screening the BILs by imposing drought at the reproductive stage, the CB 229 genotype was selected to study the transcriptome level changes and gene expression profiles of the leaves. The possible link between the whole genome transcriptome profiling with important physiological traits were studied.

\section{References}

Atlin, G. N, M. Laza, M. Amante and H. R. Lafitte. 2004. Agronomic performances of tropical aerobic, irrigated, and traditional upland rice varieties in three hydrological environments at IRRI, in New directions for a diverse planet: Proceedings of the 4th International Crop Sci Congress, Ed by Fisher T, Turner N, Angus J, McIntyre L, Robertson M, Borrell A and Lloyd D, Brisbane, Australia.

Araus, J. L., G. A. Slafer, M. P. Reynolds and C. Royo. 2002. Plant breeding and drought in C3 cereals: what should we breed for. Ann. Bot., 89: 925-940.

Baghyalakshmi K., P. Jeyaprakash, S. Ramchander, M. Raveendran and S. Robin.2016. Determination of stress indices for selection of superior genotypes under drought situation in rice (Oryza sativa L.) Int. J. Agric. Sci. 8(38). 1791-1795

Barrs, H.D. and Weatherley, P.E. 1962. A reexamination of the relative turgidity technique for estimating water deficit in leaves. Australian Journal of Biological Sciences 15, 413-428.

Beena, R., Thandapani and R. Chandrababu. 2012. Physio-morphological and biochemical characterization of selected recombinant inbred lines of rice for drought resistance. Indian $J$. Plant Physiol., 17(2): 189-193.

Bradford, M. M. 1976. A rapid and sensitive method for the quantitation of microgram quantities of protein utilizing the principle of proteindye binding. Anal. Biochem., 72: 248254.

Clarke, D. D. 1982. The accumulation of 
cinnamic acid amides in the cell walls of potato tissue as an early response to fungal attack. In Active Defense Mechanisms in Plants, ed. R. K. S. Wood, pp. 321-22. New York: Plenum.

Hao, W and H.X. Lin, 2010. Toward understanding genetic mechanisms of complex traits in rice. J Genet. Genomics., 37:653-666.

Hirasawa, T., S. Ozawa, RD. Tayraran and T. Ookawa. 2010. Varietal differences in photosynthetic rates in rice plants with special reference to the nitrogen content of leaves. Plant Prod. Sci., 13: 53-57.

Hsiao, TC and LK. Xu. 2000. Sensitivity of growth of roots versus leaves to water stress: biophysical analysis and relation to water transport. J Exp. Bot., 51: 1595-616.

Jha, B. N and R. A. Singh. 1997. Physiological responses of rice varieties to different levels of moisture stress. Indian J Plant Physiol., 2: 8184.

Lawlor, D. W and G. Cornic. 2002. Photosynthetic carbon assimilation and associated metabolism in relation to water deficits in higher plants. Plant Cell Environ., 25, 275-294.

Liu, H.Y., G. Zou, G. Liu, S. Hu, M. Li, , X. Yu, H. Mei and L. Luo. 2005. Correlation analysis and QTL identification for canopy temperature, leaf water potential and spikelet fertility in rice under contrasting moisture regimes. Chin. Sci. Bull., 50: 317-326.

Martinez, J. P., H. Silva, J. F. Ledent and M. Pinto. 2007. Effect of drought stress on the osmotic adjustment, cell wall elasticity and cell volume of six cultivars of common beans (Phaseolus vulgaris L.). Euro. J. Agro., 26: 30-38.

Millan, T., H.J. Clarke, K.H.M. Siddique, H.K. Buhariwala, P.M. Gaur, J.K. Kumar, J. Gil, G. Khal and P. Winter. 2006. Chickpea molecular breeding: New tools and concepts. Euphytica
147: 81-103.

Molden, D., T. Oweis, P. Steduto, P. Bindraban, M. A. Hanjra and J. Kijne, 2010. Improving agricultural water productivity: Between optimism and caution. Agri. Water Manag., 97: 528- 535.

O'Toole J.C., R.T. Cruz. 1980. Response of leaf water potential, stomatal reistance, and leaf rolling to water stress. Plant Physiol 65: 428432

Park, J.R., I. McFarlane, R.H. Phipps and G.Ceddia. 2011. The role of transgenic crops in sustainable development. Plant Biotechnol $\mathrm{J} ; 9: 2-21$.

Rahman, M. T., M. T. Islam and M. O. Islam. 2002. Effect of water stress at different growth stages on yield and yield contributing characters of transplanted Aman rice. Pak. J. Biol. Sci., 5:169-72.

Sikuku, P. A., G. W. Netondo, J. C., Onyango and D. M. Musyimi. 2010. Effects of water deficit on physiology and Morphology of three varieties of NERICA Rainfed rice (Oryza sativa L.). ARPN J Agri. Biol. Sci., 5: 1.

Tezara, W., V. Mitchel, S.P. Driscul and D.W. Lawlor. 2002. Effects of water deficit and its interaction with $\mathrm{CO} 2$ supply on the biochemistry and physiology of photosynthesis in sunflower. J. Exp. Bot., 53: 1781-1791.

Venuprasad, R., H. R. Lafitte and G.N. Atlin. 2007. Response to direct selection for grain yield under drought stress in rice. Crop Sci., 47, 285-293.

Vijayalakshmi, C. and M. Nagarajan. 1994. Effect of rooting pattern in rice productivity under different water regimes. J. Agron. Crop Sci., 173: 113-117.

Weatherley, P.E. 1950. Studies in the water relations of the cotton plant. I. The field measurement of water deficits in leaves. New Phytologist 49: 81-97.

\section{How to cite this article:}

Baghyalakshmi, K., P. Jeyaprakash, S. Ramchander, T. Radhamani and Raveendran, M. 2018. Comparative Study on Backcross Inbred Lines of IR64 Rice (Oryza sativa L.) Introgressed with Drought QTLs under Varied Moisture Regimes over Different Seasons. Int.J.Curr.Microbiol.App.Sci. 7(01): 2716-2725. doi: https://doi.org/10.20546/ijcmas.2018.701.325 